\title{
Material instabilities in nematic elastomers
}

\author{
Antonio DeSimone ${ }^{\mathrm{a}, *}$, Georg Dolzmann ${ }^{\mathrm{b}, 1}$ \\ ${ }^{a}$ Max Planck Institute for Mathematics in the Sciences, Inselstrasse 22-26, D-04103 Leipzig, Germany \\ ${ }^{\mathrm{b}}$ Division of Engineering and Applied Science, California Institute of Technology, Pasadena, CA 91125, USA \\ Received 16 December 1998; accepted 15 June 1999 \\ Communicated by J.M. Ball
}

\begin{abstract}
Soft deformation paths and domain patterns in nematic elastomers are analyzed through the minimization of a nonconvex free-energy recently proposed in the literature. The free-energy density has multiple wells, and is not restricted to small deformations. The problems of calculating the quasiconvex hull of the energy wells and the quasiconvex envelope of the free-energy density are formulated and solved (the latter only in two spatial dimensions). This leads to a complete characterization of the set of soft deformations paths available to a given material, and of its effective macroscopic energy. (C2000 Elsevier Science B.V. All rights reserved.
\end{abstract}

Keywords: Elastomeric polymers; Liquid crystals; Calculus of variations; Domain structures; Phase transitions

\section{Introduction}

Nematic elastomers consist of networks of cross-linked liquid crystalline polymer chains. The review paper [18] discusses some of the history of their development, their properties and physical behavior, and it contains an extensive list of references. Since the cross-linked chains realize a percolating network, nematic elastomers are rubbery solids: they are elastic and the mechanism of their elastic response is entropic. Since the chains contain nematic mesogens (rigid rod-like molecules pendant to the polymeric backbone chain: this is the case of side chain nematics to which we will confine our attention), nematic elastomers are optically uniaxial below the nematic transition temperature: at each point of the sample, the common local orientation of the aligned nematic mesogens singles out a distinguished optical axis.

Contrary to nematic liquids, however, the orientational degrees of freedom of the mesogens are coupled to the translational degrees of freedom of an underlying elastic solid (the rubbery polymer network). This coupling makes nematic elastomers very interesting as a model physical system, and it is also at the root of their potential

\footnotetext{
* Corresponding author.

E-mail addresses: desimone@mis.mpg.de (A. DeSimone), dolzmann@cco.caltech.edu (G. Dolzmann)

${ }^{1}$ On leave from Max Planck Institute for Mathematics in the Sciences, Inselstrasse 22-26, D-04103 Leipzig, Germany
} 


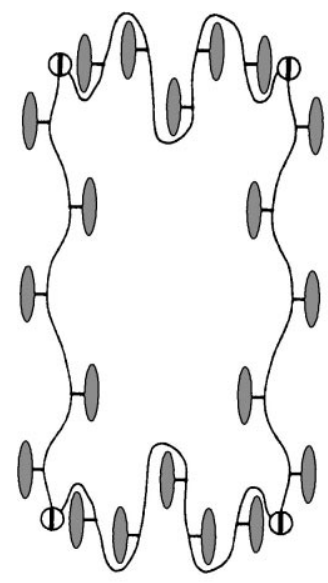

(a)

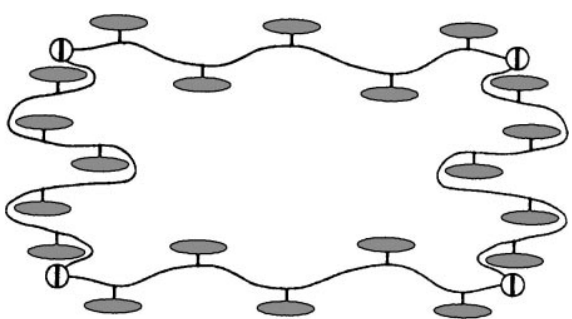

(b)

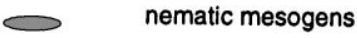

(1) crosslinks

Fig. 1. A schematic illustration of the coupling mechanism between mesogen orientation and minimum energy states of distortion of the polymer network (backbone anisotropy parameter $r>1$ ): the chains stretch in the direction along which the aligned elongated mesogens need more room. The deformation of the cross-links from the configuration (a) to the configuration (b) is accommodated by mesogen reorientations at no energy cost.

technological interest. Indeed, on one hand, orientational thermal fluctuations are hindered, and the material is optically transparent. On the other hand, the direction of the distinguished optical axis is tunable by imposing suitable strains. However, the envisaged applications (light-guiding substrates for integrated optics, bifocal contact lenses) have not (yet?) reached the development stage. Another speculative application, making no use of special optical properties, is to artificial muscles [10].

Nematic elastomers display interesting material instabilities. These are a byproduct of the symmetry breaking transformation occurring with the establishment of the orientational order characteristic of the nematic phase. At high temperatures, the nematic mesogens are randomly oriented due to thermal fluctuations, and nematic elastomers behave like isotropic rubbers. Upon cooling through the nematic transition temperature, the nematic mesogens align and the rubber network deforms uniaxially, as dictated by symmetry, with distinguished axis parallel to the common direction of the mesogens. Thus, there exists a continuum of energetically equivalent preferred states of distortion for the network (one for each direction on the unit sphere parallel to which the mesogens may align). These states may coexist in a sample, giving rise to domain patterns observable under polarized light (regions where the mesogens are differently oriented may appear opaque or transparent when observed under crossed polarizers). Moreover, they allow nematic elastomers to respond to imposed macroscopic deformations with negligible internal stress, whenever the imposed strains may be accommodated by simply reorienting the nematic mesogens (see Fig. 1). Both the occurrence of domains, in a characteristic striped texture ${ }^{2}$, and the existence of "soft" deformation modes have been observed experimentally [11]. An expression for the system's free-energy has been proposed, and it has been shown how the observed phenomena can be interpreted as attempts of the system to minimize the proposed (nonconvex) free-energy [18].

\footnotetext{
${ }^{2}$ Striped domain patterns are also observed in liquid nematics. In this case, however, they arise through a different physical mechanism, namely, convective instabilities under applied electric fields, see [7].
} 


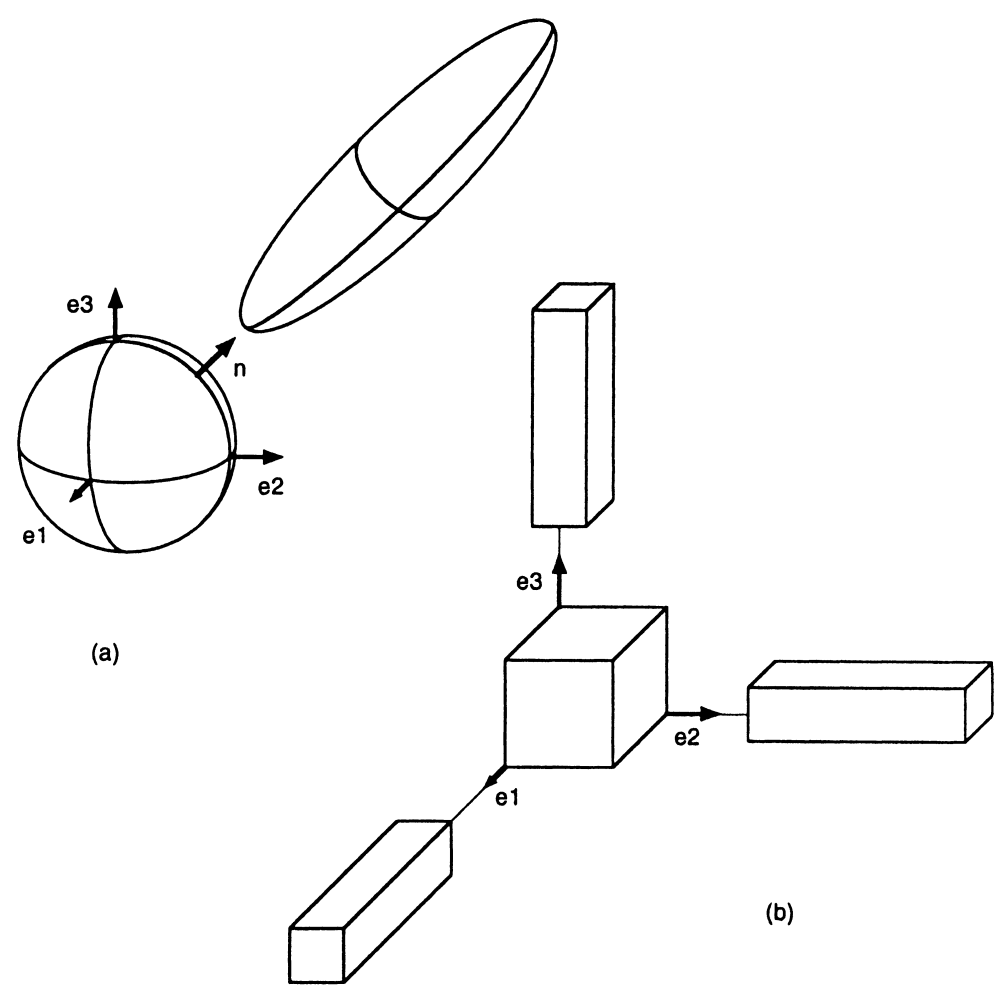

Fig. 2. Strains in two symmetry breaking transformations: (a) isotropic to uniaxial (backbone anisotropy parameter $r>1$ ), (b) cubic to tetragonal.

Our interest in nematic elastomers stems from the striking analogies that can be drawn between their mechanical behavior and that of crystalline solids undergoing martensitic phase transformations. The latter exhibit domains and soft deformations modes just like the former, and through similar mechanisms (rearrangement of twinned martensitic variants). For crystalline solids, the symmetry breaking transformations of interest involve parent phases with discrete crystallographic symmetry, and they deliver only a finite number of distinct product phases. For a cubic to tetragonal transformation, for example, there are three preferred states of distortions describing the product phases, corresponding to three uniaxial stretches $\mathbf{U}_{\mathbf{n}_{i}}, i=1,2,3$, along unit vectors $\mathbf{n}_{i}$ parallel to the edges of the cubic cell of the parent phase (see Fig. 2). Since superimposed rigid body rotations leave the energy invariant, each of the three uniaxial stretches defines an energy "well", and the stress-free (or "natural") states of the product phase are defined by the union of these wells, namely, by a set of the form $\mathbb{K}=\cup_{i=1}^{3} S O(3) \mathbf{U}_{\mathbf{n}_{i}}$, where $\mathrm{SO}(3)$ is the group of rotations in $\mathbb{R}^{3}$. Viewed from this perspective, nematic elastomers provide us with an infinite dimensional version of the last formula, namely, $\mathbb{K}=\cup_{\mathbf{n} \in S^{2}} \mathrm{SO}(3) \mathbf{U}_{\mathbf{n}}$, where $S^{2}$ is the unit sphere in $\mathbb{R}^{3}$.

There is a well-developed mathematical literature on variational approaches to phase transitions in crystalline solids (see, e.g., [2] or the lecture notes [12]). Many relevant questions on the mechanical response of materials described by multiwell energies can thus be given a precise mathematical formulation. In particular, given a material with its characteristic set of stress-free states $\mathbb{K}$, a natural question is to identify all the macroscopic deformations that can be accommodated by states belonging to $\mathbb{K}$. This set of soft macroscopic deformations turns out to be a generalized convex hull of the set $\mathbb{K}$, its quasiconvex hull $\mathbb{K}^{\mathrm{qc}}$. More generally, one may ask what is the relaxed (or effective) energy of the system corresponding to an arbitrary imposed deformation. That is, what is the energy cost of imposing an affine deformation at the boundary of a sample, while the system is allowed to relax in the interior, e.g., by developing internal domain structures if this is energetically advantageous. If $W$ is the system's 
free-energy, then $\mathbb{K}$ is its zero level set (note that whenever the latter is nonconvex, as in the multiwell examples above, the former is nonconvex). The relaxed free-energy of the system is then a generalized convex envelope of $W$, its quasiconvex envelope $W^{\text {qc }}$, whose zero level set is $\mathbb{K}^{\text {qc }}$. Given $W$ or $\mathbb{K}$, it is usually a very difficult task to compute $W^{\mathrm{qc}}$ or $\mathbb{K}^{\mathrm{qc}}$. The goal of this paper is to perform such computations for the case of nematic elastomers. The reason for our partial success is precisely the high symmetry of the isotropic parent phase.

\section{Free-energy and energy wells}

Within a continuum theory, nematic elastomers can be described using two state variables: a tensor field $\mathbf{F}$, the deformation gradient, and a vector field $\mathbf{n}$, the director. ${ }^{3}$ The deformation gradient must satisfy a kinematic compatibility condition: it must be the gradient of a continuous function $\mathbf{y}$ which maps the reference configuration $\Omega$ into the deformed configuration $\mathbf{y}(\Omega)$. Thus,

$$
\mathbf{F}(\mathbf{x})=\nabla \mathbf{y}(\mathbf{x}), \quad \mathbf{x} \in \Omega,
$$

for some continuous $\mathbf{y}$. Typically, the forces necessary to produce volume changes are much larger than those required to impose shears, leading to the customary assumption of incompressibility:

$$
\operatorname{det} \mathbf{F}(\mathbf{x})=1, \quad \mathbf{x} \in \Omega .
$$

The director field $\mathbf{n}$ is a unit vector field,

$$
|\mathbf{n}(\mathbf{x})|=1, \quad \mathbf{x} \in \Omega,
$$

describing at each point of the body the average orientation of the nematic mesogens pendant to the polymeric chains. Here we are assuming that the specimen under consideration is at a fixed temperature, lower than the isotropic to nematic transition temperature. Bladon et al. [3] have derived an expression for the free-energy of nematic elastomers by modeling them as cross-linked networks of anisotropic gaussian chains. This expression can be reduced to 4

$$
E(\mathbf{F}, \mathbf{n})=\frac{\mu}{2}\left(r^{1 / 3}\left[\operatorname{tr}\left(\mathbf{F F}^{\mathrm{T}}\right)-\frac{r-1}{r}\left(\mathbf{F F}^{\mathrm{T}}\right) \mathbf{n} \cdot \mathbf{n}\right]-3\right) .
$$

Here $\mathbf{F}^{\mathrm{T}}$ denotes the transpose of $\mathbf{F}$, tr stands for trace, while $\mu$ and $r$ are two positive material constants, respectively, the rubber energy scale (i.e., the shear modulus corresponding to $r=1$ ) and the backbone anisotropy parameter (i.e., the mean ratio of chain dimensions in the directions parallel and perpendicular to the director: for $r=1$ the chains are spherical random coils). Note that for $r=1$, the value of the backbone anisotropy parameter above the nematic transition temperature, (2.1) becomes the standard (neo-hookean) isotropic free-energy density, a function of the deformation gradient only, which is minimized at $\mathbf{F}=\mathbf{I}$, the identity tensor.

For $r \neq 1$, however, the situation is more interesting: ${ }^{5}$ let us examine first the case of a prolate backbone, i.e., $r>1$. Observe that

$$
\min _{|\mathbf{n}|=1} E(\mathbf{F}, \mathbf{n})=\frac{\mu}{2}\left(r^{1 / 3}\left[\lambda_{1}^{2}+\lambda_{2}^{2}+\lambda_{3}^{2}-\frac{r-1}{r} \max \left\{\lambda_{1}^{2}, \lambda_{2}^{2}, \lambda_{3}^{2}\right\}\right]-3\right),
$$

\footnotetext{
${ }^{3}$ In fact, a more cautious choice would be to use a traceless tensor field, the order tensor. For a uniaxial order tensor, the director describes the orientation of the distinguished axis. This level of generality will prove sufficient for the purposes of our paper.

${ }^{4}$ As in [8], we simply perform an affine change of variables, amounting to assume as reference the stress-free configuration of the isotropic parent phase rather than one of the stress-free configurations of the uniaxial product phase. This choice of a "virtual" reference configuration makes the expression for the free-energy more symmetric, and easier to analyze.

${ }^{5}$ In fact, this will lead us to a scenario already envisaged in $[13,16]$.
} 


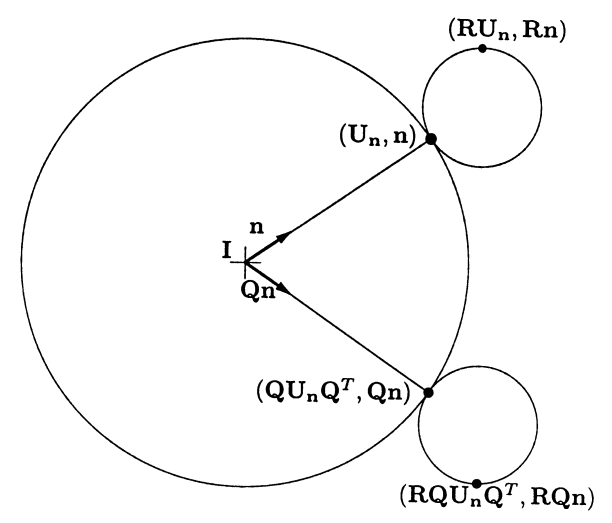

Fig. 3. A sketch of the energy wells for nematic elastomers. $\mathbf{R}$ and $\mathbf{Q}$ are arbitrary rotations.

where $\lambda_{1}^{2}, \lambda_{2}^{2}, \lambda_{3}^{2}$ are the eigenvalues of $\mathbf{F F}^{\mathrm{T}}$, is attained by taking $\mathbf{n}$ as the eigenvector of $\mathbf{F F}^{\mathrm{T}}$ corresponding to its largest eigenvalue. Furthermore, using the classical inequality between arithmetic and geometric mean, we get that

$$
\min _{\operatorname{det} \mathbf{F}=1,|\mathbf{n}|=1} E(\mathbf{F}, \mathbf{n})=\min _{\operatorname{det} \mathbf{F}=1}\left(\min _{|\mathbf{n}|=1} E(\mathbf{F}, \mathbf{n})\right)=0,
$$

which is attained by taking, say,

$$
\frac{1}{r} \lambda_{1}^{2}=\lambda_{2}^{2}=\lambda_{3}^{2}=r^{-1 / 3}
$$

Summarizing, every pair $\left(\mathbf{U}_{\mathbf{n}}, \mathbf{n}\right)$, where $\mathbf{n}$ is a unit vector and

$$
\mathbf{U}_{\mathbf{n}}=r^{1 / 3} \mathbf{n} \otimes \mathbf{n}+r^{-1 / 6}(\mathbf{I}-\mathbf{n} \otimes \mathbf{n})
$$

(here $\otimes$ denotes the tensor product of two vectors), is a minimizer of $E$. Note that the deformation described by $\mathbf{U}_{\mathbf{n}}$ is the one that maps a ball of radius 1 onto a prolate spheroid with major axis $r^{1 / 3}$ parallel to $\mathbf{n}$. Since, by frame indifference, $E(\mathbf{R F}, \mathbf{R n})=E(\mathbf{F}, \mathbf{n})$ for every rotation $\mathbf{R}$, the energy wells of the material at hand can be described by the formula

$$
\mathbb{K}=\bigcup_{\mathbf{n} \in S^{2}} \operatorname{SO}(3)\left(\mathbf{U}_{\mathbf{n}}, \mathbf{n}\right),
$$

where $S^{2}$ is the sphere of radius 1 describing the unit vectors of $\mathbb{R}^{3}$, while $\mathrm{SO}(3)$ is the group of proper rotations in $\mathbb{R}^{3}$. Fig. 3 gives a schematic description of the set $\mathbb{K}$.

The case $r<1$ (oblate backbone) is analyzed in a similar fashion: (2.2) becomes

$$
\min _{|\mathbf{n}|=1} E(\mathbf{F}, \mathbf{n})=\frac{\mu}{2}\left(r^{1 / 3}\left[\lambda_{1}^{2}+\lambda_{2}^{2}+\lambda_{3}^{2}+\frac{1-r}{r} \min \left\{\lambda_{1}^{2}, \lambda_{2}^{2}, \lambda_{3}^{2}\right\}\right]-3\right),
$$

and the minimizers of $E$ have again the structure (2.4), but in this case $\mathbf{U}_{\mathbf{n}}$ maps the ball of radius 1 onto an oblate spheroid, with minor axis $r^{1 / 3}$ parallel to $\mathbf{n}$. In the remainder of the paper, our analysis will focus on the energies

$$
\begin{aligned}
& W(\mathbf{F}):=\frac{\mu}{2}\left(r^{1 / 3}\left[\operatorname{tr}\left(\mathbf{F F}^{\mathrm{T}}\right)-\frac{r-1}{r} \max _{|\mathbf{n}|=1}\left|\mathbf{F}^{\mathrm{T}} \mathbf{n}\right|^{2}\right]-3\right), \quad r>1, \\
& W_{\mathrm{ob}}(\mathbf{F}):=\frac{\mu}{2}\left(r^{1 / 3}\left[\operatorname{tr}\left(\mathbf{F F}^{\mathrm{T}}\right)+\frac{1-r}{r} \min _{|\mathbf{n}|=1}\left|\mathbf{F}^{\mathrm{T}} \mathbf{n}\right|^{2}\right]-3\right), \quad 0<r<1,
\end{aligned}
$$


obtained from $E$ by minimizing out the director. ${ }^{6}$ This amounts to assuming that no physical mechanism hinders the director from relaxing, at each point $\mathbf{x}$ of $\Omega$, to the minimum energy state compatible with the local deformation $\mathbf{F}(\mathbf{x})$.

\section{Soft deformations}

Consider a sheet of nematic elastomer parallel to the $y z$ plane, which is initially in the zero energy configuration described by $\mathbf{F}_{0}=\mathbf{U}_{\mathbf{e}_{3}}, \mathbf{n}_{0}=\mathbf{e}_{3}$ (here $\mathbf{e}_{1}, \mathbf{e}_{2}, \mathbf{e}_{3}$ are the three orthonormal vectors parallel to the $x y z$ axes of a given right-handed cartesian frame), and assume that the specimen is stretched along the y axis, so that the macroscopic deformation of the sheet is

$$
\mathbf{F}_{t}=\left[\begin{array}{ccc}
r^{-1 / 6} & 0 & 0 \\
0 & \lambda(t) & 0 \\
0 & 0 & r^{1 / 6} / \lambda(t)
\end{array}\right], \quad r^{-1 / 6}=\lambda(0) \leq \lambda(t) \leq \lambda(1)=r^{1 / 3},
$$

with $\lambda(t)$ an increasing function of $t \in[0,1]$. Here, we are assuming $r>1$. Clearly, with the definition above, $\mathbf{F}_{1}=\mathbf{U}_{\mathbf{e}_{2}}$. Since for $t \neq 0,1$ the eigenvalues of $\mathbf{F}_{t} \mathbf{F}_{t}^{\mathrm{T}}$ do not satisfy (2.3), the uniform deformation with gradient $\mathbf{F}_{t}$ has positive energy. However, for $\delta^{2}(t)=(1 / \lambda(t))\left(\lambda^{2}(t)-r^{-1 / 3}\right)\left(r^{2 / 3}-\lambda^{2}(t)\right)$, the tensors

$$
\mathbf{F}_{t}^{ \pm}=\left[\begin{array}{ccc}
r^{-1 / 6} & 0 & 0 \\
0 & \lambda(t) & \pm \delta(t) \\
0 & 0 & r^{1 / 6} / \lambda(t)
\end{array}\right], \quad \mathbf{F}_{t}^{+}-\mathbf{F}_{t}^{-}=2 \delta(t) \mathbf{e}_{2} \otimes \mathbf{e}_{3}
$$

satisfy the eigenvalue conditions guaranteeing that $\mathbf{F}_{t}^{ \pm} \in \mathbb{K}_{\mathrm{el}}$. Therefore, if $\mathbf{n}_{t}^{ \pm}$is the eigenvector corresponding to the maximum eigenvalue of $\left(\mathbf{F}_{t}^{ \pm}\right)\left(\mathbf{F}_{t}^{ \pm}\right)^{\mathrm{T}}$, the pairs $\left(\mathbf{F}_{t}^{+}, \mathbf{n}_{t}^{+}\right),\left(\mathbf{F}_{t}^{-}, \mathbf{n}_{t}^{-}\right)$lie on the energy wells. In addition, as shown by the second formula in (3.2), they differ by the rank-one tensor $2 \delta(t) \mathbf{e}_{2} \otimes \mathbf{e}_{3}$. It follows that we can in fact construct a continuous deformation whose gradient takes the values $\mathbf{F}_{t}^{+}, \mathbf{F}_{t}^{-}$on stripes orthogonal to $\mathbf{e}_{3}$. Indeed, for $k$ integer, and denoting by $\chi$ the one-periodic function taking the value zero in $\left[0, \frac{1}{2}\right)$ and the value one in $\left[\frac{1}{2}, 1\right)$, the deformation

$$
\mathbf{y}^{k}(\mathbf{x})=\mathbf{F}_{t}^{-} \mathbf{x}+\frac{2 \delta(t)}{k} \mathbf{e}_{2} \int_{0}^{k \mathbf{x} \cdot \mathrm{e}_{3}} \chi(s) \mathrm{d} s
$$

has the required properties. Note that $1 / k$ is proportional to the width of the stripes, and as $k \rightarrow \infty$, $\mathbf{y}^{k}$ converges uniformly to the affine deformation

$$
\mathbf{y}(\mathbf{x})=\left(\frac{1}{2} \mathbf{F}_{t}^{-}+\frac{1}{2} \mathbf{F}_{t}^{+}\right) \mathbf{x}=\mathbf{F}_{t} \mathbf{x}
$$

where the factors $\frac{1}{2}$ arise from the fact that $\mathbf{F}_{t}^{+}$and $\mathbf{F}_{t}^{-}$occupy the same volume fraction (namely, $\frac{1}{2}$ ) of the specimen.

The discussion above summarizes Warner and Terentjev's interpretation [18] of the experimental observation of striped domain patterns by Kundler and Finkelmann [11]. From the energetic viewpoint, the key fact is that for $t \neq 0,1$ the free-energy $W$ is not convex along the rank-one segment that joins $\mathbf{F}_{t}^{+}$and $\mathbf{F}_{t}^{-}$in matrix space. In the midpoint of this segment

$$
\mathbf{F}_{t}=\mathbf{F}_{t}^{-}+\frac{1}{2}\left(\mathbf{F}_{t}^{+}-\mathbf{F}_{t}^{-}\right)
$$

\footnotetext{
${ }^{6}$ Note that both (2.6) and (2.7) are isotropic, see (A.2), and that their energy wells are of the form $\mathbb{K}_{\mathrm{el}}=\cup_{\mathbf{n} \in S^{2}} \mathrm{SO}(3) \mathbf{U}_{\mathbf{n}}$. Starting from Section 4 , we will simplify the notation by writing $\mathbb{K}$, rather than $\mathbb{K}_{\mathrm{el}}$, for the set of energy wells of (2.6) and (2.7).
} 

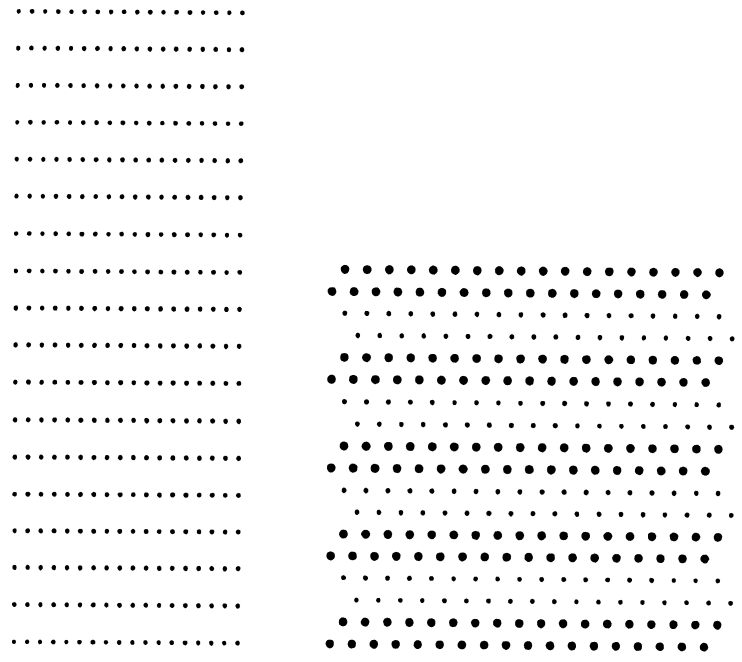

Fig. 4. The observed domain patterns resolving the soft deformation path (3.1) from $\mathbf{U}_{\mathbf{e}_{3}}$ (top left) to $\mathbf{U}_{\mathbf{e}_{2}}$ (bottom right). Here, as in the picture below, the difference in the size of the dots hints at the different optical contrast induced in polarized light by the director.

we have

$$
W\left(\mathbf{F}_{t}^{-}+\frac{1}{2}\left(\mathbf{F}_{t}^{+}-\mathbf{F}_{t}^{-}\right)\right)>W\left(\mathbf{F}_{t}^{-}\right)+\frac{1}{2}\left(W\left(\mathbf{F}_{t}^{+}\right)-W\left(\mathbf{F}_{t}^{-}\right)\right)=0
$$

Thus, it is always energetically advantageous to replace the uniform macroscopic deformation $\mathbf{F}_{t}$ with a nonuniform (mesoscopic) domain pattern with macroscopic average $\mathbf{F}_{t}$, but such that the deformation gradients are locally $\mathbf{F}_{t}^{-}$ and $\mathbf{F}_{t}^{+}$. As $\lambda(t)$ is increased from $r^{-1 / 6}$ to $r^{1 / 3}$, the sheet may follow a zero-energy path (hence a path along which the system could, in principle, evolve without developing internal stresses) joining the states $\left(\mathbf{U}_{\mathbf{e}_{3}}, \mathbf{e}_{3}\right)$ and $\left(\mathbf{U}_{\mathbf{e}_{2}}, \mathbf{e}_{2}\right)$, finally resulting in the macroscopic stretch $r^{1 / 3} / r^{-1 / 6}=\sqrt{r}$ along the $y$ axis (see Fig. 4).

\section{Quasiconvexity: motivation}

The energetic interpretation of the emergence of domain patterns discussed in the previous section is based on the fact that a system may find an energetic advantage in letting its state variables develop fine scale spatial oscillations, while their macroscopic averages are held fixed. One may ask oneself for conditions on the free-energy of the system which lead to or forbid this kind of behavior. In particular, one may ask which convexity properties of the free-energy, say $\Phi \geq 0$, guarantee that a uniform deformation is always the energy minimizer under its own boundary conditions:

$$
\Phi(\mathbf{F}) \leq \frac{1}{\operatorname{volume}(\Omega)} \int_{\Omega} \Phi(\mathbf{F}+\nabla \mathbf{u}(\mathbf{x})) \mathrm{d} \mathbf{x},
$$


where $\mathbf{u}(\mathbf{x})$ is a smooth perturbation of the affine deformation $\mathbf{F x}$, vanishing on the boundary of $\Omega$, but otherwise arbitrary. A function $\Phi$ that satisfies the inequality above is called quasiconvex. This property is 'intermediate' between the weaker notion of rank-one convexity (the property of being convex along rank-one lines), and the stronger notion of polyconvexity (a polyconvex function of $\mathbf{F}$ is a convex function of the minors of $\mathbf{F}$ ). Convexity is even stronger, but all these notions collapse onto the same one when the state variable is a scalar or a vector. Although quasiconvexity seems the property more closely linked to physical intuition, it is the hardest to check. On the contrary, it is relatively easy to check for rank-one convexity or polyconvexity, but there is a gap between these two notions ${ }^{7}$, and quasiconvexity sits in the middle. Interestingly, with nematic elastomers we jump from one to the other side of this gap when crossing the nematic transition temperature. At low temperatures, i.e., for $r \neq 1$, Eq. (3.3) shows that the free-energy $W$ fails to be rank-one convex: indeed we can lower the system's energy by layering along rank-one lines. In the high temperature isotropic phase $(r=1)$ the free-energy $W$ is polyconvex ${ }^{8}$, hence quasiconvex, and uniform deformations prevail.

Assume now that $\mathbf{F}$ is a deformation gradient for which (4.1) fails. Then $\Phi(\mathbf{F})$ gives little information about the energy cost (per unit volume) required to enforce the macroscopic deformation $\mathbf{F}$. Rather, the infimum

$$
\inf _{\mathbf{u}}\left\{\frac{1}{\operatorname{volume}(\Omega)} \int_{\Omega} \Phi(\mathbf{F}+\nabla \mathbf{u}(\mathbf{x})) \mathrm{d} \mathbf{x}\right\}
$$

is the physically relevant quantity, and the only one experimentally accessible with a macroscopic measurement (of course, provided that energy minimization is the physically relevant mechanism for the material's response). Thus, we refer to (4.2) as the relaxed or effective energy of the system. It turns out that (4.2) is equal to $\Phi^{\mathrm{qc}}(\mathbf{F})$, the quasiconvex envelope of $\Phi$ (see, e.g., [12]). Moreover, if $\mathbb{K}$ is the zero level set of $\Phi$, then the zero level set of $\Phi^{\mathrm{qc}}(\mathbf{F})$ is $\mathbb{K}^{\mathrm{qc}}$, the quasiconvex hull of $\mathbb{K}$. Thus $\mathbb{K}^{\mathrm{qc}}$ is the set of macroscopic deformations that can be imposed at zero energy cost, i.e., the soft deformations. Any curve contained in $\mathbb{K}^{\mathrm{qc}}$ defines then a soft deformation path, i.e., a path along which the system may (in principle) evolve without developing internal stresses.

In the next two sections, we turn to the actual calculation of the relaxed energy $W^{\text {qc }}$ (for a two-dimensional version of $W$, suitable to describe plane strain geometries) and of the set of soft deformations $\mathbb{K}^{\mathrm{qc}}$ for nematic elastomers. For this purpose, it proves expedient to rewrite (2.6) and (2.7) in a more manageable form. For $\mathbf{F} \in \mathbb{M}^{n \times n}$, $\operatorname{det} \mathbf{F}=1$, let $\lambda_{1}^{2} \geq \lambda_{2}^{2} \geq \cdots \geq \lambda_{n}^{2}$ be the ordered eigenvalues of $\mathbf{F F}^{\mathrm{T}}$. Then (2.6) and (2.7) can be written as

$$
\begin{aligned}
& W(\mathbf{F})=(1-\epsilon) \lambda_{1}^{2}+\lambda_{2}^{2}+\cdots+\lambda_{n}^{2}-n(1-\epsilon)^{1 / n}, \quad 0<\epsilon<1, \\
& W_{\mathrm{ob}}(\mathbf{F})=\lambda_{1}^{2}+\lambda_{2}^{2}+\cdots+(1+\bar{\epsilon}) \lambda_{n}^{2}-n(1+\bar{\epsilon})^{1 / n}, \quad 0<\bar{\epsilon},
\end{aligned}
$$

where we have set $\epsilon=(r-1) / r$ for $r>1, \bar{\epsilon}=(1-r) / r$ for $0<r<1$, and we have normalized to one the multiplicative constant $(\mu / 2)(r)^{1 / n}$ (this is done without loss of generality since clearly $(\lambda W)^{\mathrm{qc}}=\lambda W^{\mathrm{qc}}, \forall \lambda \geq 0$ ). Using, as before, the inequality between the arithmetic and the geometric mean we obtain that

$$
\begin{aligned}
& \lambda_{1}^{2}=(1-\epsilon)^{1 / n-1}>1, \quad \lambda_{2}^{2}=\cdots=\lambda_{n}^{2}=(1-\epsilon)^{1 / n}<1, \\
& \lambda_{1}^{2}=\cdots=\lambda_{n-1}^{2}=(1+\bar{\epsilon})^{1 / n}>1, \quad \lambda_{n}^{2}=(1+\bar{\epsilon})^{1 / n-1}<1
\end{aligned}
$$

minimize, respectively, (4.3) and (4.4) under the constraint $\operatorname{det} \mathbf{F}=1$ at the value zero.

\footnotetext{
${ }^{7}$ See [15]. The interested reader should, at this point, refer to Appendix A for a summary of definitions and main results related to convexity properties of free-energy densities that are used in the sequel.

${ }^{8}$ By Lemma A.2, convexity fails due to the incompressibility constraint.
} 


\section{A two-dimensional model problem}

For $n=2$ the free-energies (4.3) and (4.4) become

$$
\begin{aligned}
& W(\mathbf{F})=(1-\epsilon) \max \left\{\lambda_{1}^{2}, \lambda_{2}^{2}\right\}+\min \left\{\lambda_{1}^{2}, \lambda_{2}^{2}\right\}-2 \sqrt{1-\epsilon}, \\
& W_{\mathrm{ob}}(\mathbf{F})=\max \left\{\lambda_{1}^{2}, \lambda_{2}^{2}\right\}+(1+\bar{\epsilon}) \min \left\{\lambda_{1}^{2}, \lambda_{2}^{2}\right\}-2 \sqrt{1+\bar{\epsilon}},
\end{aligned}
$$

which, for $\lambda_{1} \lambda_{2}=1$, are minimized at the value zero by taking, respectively,

$$
\begin{aligned}
& \max \left\{\lambda_{1}, \lambda_{2}\right\}=(1-\epsilon)^{-1 / 4}>1, \quad \min \left\{\lambda_{1}, \lambda_{2}\right\}=(1-\epsilon)^{1 / 4}<1, \\
& \max \left\{\lambda_{1}, \lambda_{2}\right\}=(1+\bar{\epsilon})^{1 / 4}>1, \quad \min \left\{\lambda_{1}, \lambda_{2}\right\}=(1+\bar{\epsilon})^{-1 / 4}<1 .
\end{aligned}
$$

Thus, the energy wells for both $W$ and $W_{\mathrm{ob}}$ can be described by the formula

$$
\mathbb{K}=\bigcup_{\mathbf{e} \in S^{1}} \operatorname{SO}(2)\left(\eta(\mathbf{e} \otimes \mathbf{e})+\frac{1}{\eta}\left(\mathbf{e}^{\perp} \otimes \mathbf{e}^{\perp}\right)\right),
$$

where, for $\mathbf{e}=(\cos \vartheta, \sin \vartheta), \mathbf{e}^{\perp}$ is the vector $(-\sin \vartheta, \cos \vartheta)$, and we take $\eta=(1-\epsilon)^{-1 / 4}$ in the prolate case, $\eta=(1+\bar{\epsilon})^{1 / 4}$ in the oblate case ${ }^{9}$.

Lemma 5.1. For $\eta>1$, let

$$
\mathbb{K}=\bigcup_{\mathbf{e} \in S^{1}} \operatorname{SO}(2)\left(\eta(\mathbf{e} \otimes \mathbf{e})+\frac{1}{\eta}\left(\mathbf{e}^{\perp} \otimes \mathbf{e}^{\perp}\right)\right) .
$$

Then $\mathbb{K}^{\mathrm{rc}}=\mathbb{K}^{\mathrm{qc}}=\mathbb{K}^{\mathrm{pc}}$ and any of these sets is given by

$$
\left\{\mathbf{F} \in \mathbb{M}^{2 \times 2}: \operatorname{det} \mathbf{F}=1, \mathbf{F} \text { has singular values } \lambda_{i} \in\left[\frac{1}{\eta}, \eta\right]\right\} \text {. }
$$

Proof. Denote by $\mathcal{A}$ the set (5.2). Let $t^{+}=\max \{0, t\}$ and define

$$
\Phi(\mathbf{F})=(\operatorname{det} \mathbf{F}-1)^{2}+\sup _{\mathbf{e} \in S^{1}}\left(|\mathbf{F e}|^{2}-\eta^{2}\right)^{+} .
$$

Then $\Phi$ is polyconvex and $\Phi(\mathbf{F})=0$ if and only if $\mathbf{F} \in \mathcal{A}$. Thus $\mathbb{K}^{\text {pc }} \subset \mathcal{A}$. We claim that $\mathcal{A} \subset \mathbb{K}^{\mathrm{rc}}$. Since $\mathbb{K}$ is invariant under orthogonal conjugation and under multiplication from the left by elements in $\mathrm{SO}(2)$ it suffices to show that diagonal matrices $\mathbf{F}(t)=\operatorname{diag}(t, 1 / t)$ belong to $\mathbb{K}^{\mathrm{rc}}$ for $t \in[1 / \eta, \eta]$. This follows from Lemma A.5 by taking $1 / \eta=a$ and $\eta=b$. We conclude $\mathbb{K}^{\mathrm{pc}} \subset \mathcal{A} \subset \mathbb{K}^{\mathrm{rc}} \subset \mathbb{K}^{\mathrm{pc}}$ and the assertion of the lemma follows.

Proposition 5.2. Let $\eta=(1-\epsilon)^{-1 / 4}$ and define $\phi: \mathbb{R}_{+}^{2} \rightarrow \mathbb{R}$ by

$$
\phi\left(\lambda_{1}, \lambda_{2}\right)= \begin{cases}(1-\epsilon) \max \left\{\lambda_{1}^{2}, \lambda_{2}^{2}\right\}+\min \left\{\lambda_{1}^{2}, \lambda_{2}^{2}\right\}-2 \sqrt{1-\epsilon} & \text { if } \lambda_{1} \lambda_{2}=1 \\ +\infty & \text { else }\end{cases}
$$

Assume that $W: \mathbb{M}^{2 \times 2} \rightarrow \mathbb{R}$ is given by $W(\mathbf{F})=\phi\left(\lambda_{1}, \lambda_{2}\right)$, where $\lambda_{1}$, and $\lambda_{2}$ are the singular values of $\mathbf{F}$. Then $W^{\mathrm{pc}}(\mathbf{F})=W^{\mathrm{qc}}(\mathbf{F})=W^{\mathrm{rc}}(\mathbf{F})=\tilde{\phi}\left(\lambda_{1}, \lambda_{2}\right)$, where

$$
\tilde{\phi}\left(\lambda_{1}, \lambda_{2}\right)= \begin{cases}\phi\left(\lambda_{1}, \lambda_{2}\right) & \text { if } \lambda_{1} \lambda_{2}=1, \max \left\{\lambda_{1}, \lambda_{2}\right\} \geq \eta, \\ 0 & \text { if } \lambda_{1} \lambda_{2}=1, \max \left\{\lambda_{1}, \lambda_{2}\right\} \in[1, \eta], \\ +\infty & \text { else. }\end{cases}
$$

\footnotetext{
${ }^{9}$ With this notation, $\eta$ is always greater than 1 and the preferred director orientation corresponding to the deformation $\mathbf{F}=\eta(\mathbf{e} \otimes \mathbf{e})+$ $(1 / \eta)\left(\mathbf{e}^{\perp} \otimes \mathbf{e}^{\perp}\right)$ is $\mathbf{e}$ in the prolate case, and $\mathbf{e}^{\perp}$ in the oblate case.
} 
Proof. Recall from (5.1) that $W(\mathbf{F})=0$ if and only if

$$
\mathbf{F} \in \mathbb{K}=\bigcup_{\mathbf{e} \in S^{1}} \operatorname{SO}(2)\left(\eta(\mathbf{e} \otimes \mathbf{e})+\frac{1}{\eta}\left(\mathbf{e}^{\perp} \otimes \mathbf{e}^{\perp}\right)\right) .
$$

Now let $\tilde{W}(\mathbf{F})=\tilde{\phi}\left(\lambda_{1}, \lambda_{2}\right)$. We will show that $W^{\mathrm{rc}} \leq \tilde{W}=\tilde{W}^{\mathrm{pc}} \leq W^{\mathrm{pc}}$. This proves the assertion since in general $W^{\mathrm{pc}} \leq W^{\mathrm{qc}} \leq W^{\mathrm{rc}}$. In order to establish the first inequality it suffices to show that $W^{\mathrm{rc}}(\mathbf{F}) \leq \tilde{W}(\mathbf{F})$ for all $\mathbf{F}$ of the form

$$
\mathbf{F}=\mathbf{R}\left(t(\mathbf{e} \otimes \mathbf{e})+\frac{1}{t}\left(\mathbf{e}^{\perp} \otimes \mathbf{e}^{\perp}\right)\right), \mathbf{R} \in \operatorname{SO}(2), \mathbf{e} \in S^{1}, t \in[1, \eta] .
$$

By Lemma A.5 this is equivalent to $\mathbf{F} \in \mathbb{K}^{\mathrm{rc}}$ and thus $W^{\mathrm{rc}}(\mathbf{F})=0=\tilde{W}(\mathbf{F})$.

It remains to prove that $\tilde{W}$ is polyconvex. Let

$$
I_{1}(t)= \begin{cases}0 & \text { if } t=1 \\ \infty & \text { else }\end{cases}
$$

Since $\tilde{W}(\mathbf{F})=\tilde{\phi}\left(\lambda_{1}, \lambda_{2}\right)+I_{1}\left(\lambda_{1} \lambda_{2}\right)$, by Lemma A. 2 it suffices to show that $\tilde{\phi}$ is the restriction to the set $\left\{\lambda_{1} \lambda_{2}=1\right\}$ of a convex function which is nondecreasing in its variables. We will prove this by constructing a convex extension $\hat{\phi}$ of $\tilde{\phi}$ which is nondecreasing in its variables. For $\left(x_{0}, y_{0}\right) \in \mathbb{R}_{+}^{2}$ with $x_{0}+y_{0} \geq 2$ we denote by $\left(t^{-}, t^{+}\right)$and $\left(t^{+}, t^{-}\right)$the two intersection points of the line $x+y=x_{0}+y_{0}$ with the hyperbola $x y=1$, where

$$
t^{ \pm}=\frac{x_{0}+y_{0}}{2} \pm \sqrt{\frac{\left(x_{0}+y_{0}\right)^{2}}{4}-1}
$$

Let $2 s(\epsilon)=(1-\epsilon)^{1 / 4}+(1-\epsilon)^{-1 / 4}$ and

$$
g(s)= \begin{cases}\phi\left(t^{-}(s, s), t^{+}(s, s)\right) & \text { if } s>s(\epsilon) \\ 0 & \text { if } s \in[0, s(\epsilon)]\end{cases}
$$

Clearly $g$ is nonnegative and continuous since $t^{+}(s(\epsilon), s(\epsilon))=\eta, t^{-}(s(\epsilon), s(\epsilon))=1 / \eta$ and $\phi(1 / \eta, \eta)=0$. We show next that $g:[0, \infty) \rightarrow \mathbb{R}$ is convex and nondecreasing. Indeed, for $s \geq s(\epsilon)>1$,

$$
g(s)=(2-\epsilon)\left(2 s^{2}-1\right)-2 \epsilon s \sqrt{s^{2}-1}-2 \sqrt{1-\epsilon}
$$

and a short calculation shows that

$$
g^{\prime \prime}(s)=8\left(1-\epsilon \frac{s^{3}-(3 / 2) s}{\left(s^{2}-1\right)^{3 / 2}}\right) \geq 0 \text { for } s>s(\epsilon)
$$

(this is because the fraction is negative for $1<s<\sqrt{3 / 2}$ and less than one for $s \geq \sqrt{3 / 2}$ ). Since $g$ is nonnegative with $g(s(\epsilon))=0,(5.3)$ shows that $g$ is convex and nondecreasing on $[0, \infty)$. Now define $\hat{\phi}: \mathbb{R}_{+}^{2} \rightarrow \mathbb{R}$ by

$$
\hat{\phi}\left(\lambda_{1}, \lambda_{2}\right)=g\left(\frac{1}{2}\left(\lambda_{1}+\lambda_{2}\right)\right)
$$

Since $g$ is convex and nondecreasing, $\hat{\phi}$ is convex on $\mathbb{R}_{+}^{2}$ and nondecreasing in its variables. Finally, since for $t>1$,

$$
t^{+}\left(\frac{1}{2}\left(t+\frac{1}{t}\right), \frac{1}{2}\left(t+\frac{1}{t}\right)\right)=t, \quad t^{-}\left(\frac{1}{2}\left(t+\frac{1}{t}\right), \frac{1}{2}\left(t+\frac{1}{t}\right)\right)=\frac{1}{t},
$$


we conclude

$$
\hat{\phi}\left(t, \frac{1}{t}\right)=g\left(\frac{1}{2}\left(t+\frac{1}{t}\right)\right)= \begin{cases}\phi(1 / t, t) & \text { if } \frac{1}{2}(t+1 / t)>s(\epsilon) \\ 0 & \text { if } \frac{1}{2}(t+1 / t) \in[0, s(\epsilon)] .\end{cases}
$$

It is easy to see that $\frac{1}{2}(t+1 / t)>s(\epsilon)$ if and only if $\max \{t, 1 / t\}>\eta$. Thus $\hat{\phi}(t, 1 / t)=\tilde{\phi}(t, 1 / t)$, i.e., $\hat{\phi}$ is the desired extension. This proves the proposition.

The last proposition enables us to compute $W_{\mathrm{ob}}^{\mathrm{qc}}$ as well. We simply observe that, defining $\epsilon(\bar{\epsilon}):=\bar{\epsilon} /(1+\bar{\epsilon})$, we may write $W_{\mathrm{ob}}$ as

$$
W_{\mathrm{ob}}(\mathbf{F})=(1+\bar{\epsilon}) \bar{\phi}\left(\lambda_{1}, \lambda_{2}\right)
$$

where

$$
\bar{\phi}\left(\lambda_{1}, \lambda_{2}\right)= \begin{cases}(1-\epsilon(\bar{\epsilon})) \max \left\{\lambda_{1}^{2}, \lambda_{2}^{2}\right\}+\min \left\{\lambda_{1}^{2}, \lambda_{2}^{2}\right\}-2 \sqrt{1-\epsilon(\bar{\epsilon})} & \text { if } \lambda_{1} \lambda_{2}=1 \\ +\infty & \text { else. }\end{cases}
$$

Thus, setting $t_{0}(\bar{\epsilon})=(1-\epsilon(\bar{\epsilon}))^{-1 / 4}$, Proposition 5.2 implies that

$$
W_{\mathrm{ob}}^{\mathrm{qc}}(\mathbf{F})= \begin{cases}(1+\bar{\epsilon}) \bar{\phi}\left(\lambda_{1}, \lambda_{2}\right) & \text { if } \lambda_{1} \lambda_{2}=1, \max \left\{\lambda_{1}, \lambda_{2}\right\} \geq t_{0}(\bar{\epsilon}), \\ 0 & \text { if } \lambda_{1} \lambda_{2}=1, \max \left\{\lambda_{1}, \lambda_{2}\right\} \in\left[1, t_{0}(\bar{\epsilon})\right], \\ +\infty & \text { else. }\end{cases}
$$

\section{The three-dimensional case}

For $n=3$, the energy wells described by (4.5) and (4.6) take the form

$$
\mathbb{K}=\bigcup_{\mathbf{e} \in S^{2}} \operatorname{SO}(3)\left(\eta^{2}(\mathbf{e} \otimes \mathbf{e})+\frac{1}{\eta}(\mathbf{I}-\mathbf{e} \otimes \mathbf{e})\right)
$$

where $\eta^{2}=(1-\epsilon)^{-1 / 3}>1$ in the prolate case, $\eta^{2}=(1+\bar{\epsilon})^{-1 / 3}<1$ in the oblate case. ${ }^{10}$

Proposition 6.1. For $\eta>0$, let

$$
\mathbb{K}=\bigcup_{\mathbf{e} \in S^{2}} \operatorname{SO}(3)\left(\eta^{2}(\mathbf{e} \otimes \mathbf{e})+\frac{1}{\eta}(\mathbf{I}-\mathbf{e} \otimes \mathbf{e})\right)
$$

Then $\mathbb{K}^{\mathrm{rc}}=\mathbb{K}^{\mathrm{qc}}=\mathbb{K}^{\mathrm{pc}}$ and any of these sets is given by

$$
\left\{\mathbf{F} \in \mathbb{M}^{3 \times 3}: \operatorname{det} \mathbf{F}=1, \mathbf{F} \text { has principal values } \lambda_{i} \in\left[\eta^{-}, \eta^{+}\right]\right\},
$$

where $\eta^{-}=\min \left\{\eta^{2}, 1 / \eta\right\}$, and $\eta^{+}=\max \left\{\eta^{2}, 1 / \eta\right\}$.

\footnotetext{
${ }^{10}$ With this notation, the preferred director orientation corresponding to the deformation $\mathbf{F}=\eta^{2}(\mathbf{e} \otimes \mathbf{e})+(1 / \eta)(\mathbf{I}-\mathbf{e} \otimes \mathbf{e})$ is $\mathbf{e}$ both in the prolate and in the oblate case.
} 
Proof. Denote by $\mathcal{A}$ the set (6.1), let $t^{+}=\max \{0, t\}$, and define

$$
\Phi(\mathbf{F})=(\operatorname{det} \mathbf{F}-1)^{2}+\sup _{\mathbf{e} \in S^{2}}\left(|\mathbf{F e}|^{2}-\left(\eta^{+}\right)^{2}\right)^{+}+\sup _{\mathbf{e} \in S^{2}}\left(|(\operatorname{cof} \mathbf{F}) \mathbf{e}|^{2}-\frac{1}{\left(\eta^{-}\right)^{2}}\right)^{+} .
$$

Then $\Phi$ is polyconvex and $\Phi(\mathbf{F})=0$ if and only if $\mathbf{F} \in \mathcal{A}$. Thus $\mathbb{K}^{\mathrm{pc}} \subset \mathcal{A}$.

We claim that $\mathcal{A} \subset \mathbb{K}^{\mathrm{rc}}$. Since $\mathbb{K}$ is invariant under orthogonal conjugation and multiplication from the left by elements in $\operatorname{SO}(3)$ it suffices to show that diagonal matrices $\operatorname{diag}\left(\lambda_{1}, \lambda_{2}, \lambda_{3}\right)$ with $\lambda_{1} \lambda_{2} \lambda_{3}=1$ and $\lambda_{i} \in\left[\eta^{-}, \eta^{+}\right]$ belong to $\mathbb{K}^{\mathrm{rc}}$. We prove this for the case $\eta>1$ (so that $\eta^{-}=1 / \eta$ and $\eta^{+}=\eta^{2}$ ) by applying Lemma A.5 several times. Denote by $\mathcal{R}_{i} \subset \mathrm{SO}(3)$ the set of all rotations about $\mathbf{e}_{i}$. Since the axis of rotation is fixed, $\mathcal{R}_{i}$ is in fact a copy of $\operatorname{SO}(2)$. For every $\lambda_{1} \in\left[1 / \eta, \eta^{2}\right]$,

$$
\begin{aligned}
& \operatorname{diag}\left(\lambda_{1}, \frac{\eta}{\lambda_{1}}, \frac{1}{\eta}\right) \in\left(\mathcal{R}_{3} \operatorname{diag}\left(\eta^{2}, \frac{1}{\eta}, \frac{1}{\eta}\right) \cup \mathcal{R}_{3} \operatorname{diag}\left(\frac{1}{\eta}, \eta^{2}, \frac{1}{\eta}\right)\right)^{\mathrm{rc}} \subset \mathbb{K}^{\mathrm{rc}}, \\
& \operatorname{diag}\left(\lambda_{1}, \frac{1}{\eta}, \frac{\eta}{\lambda_{1}}\right) \in\left(\mathcal{R}_{2} \operatorname{diag}\left(\eta^{2}, \frac{1}{\eta}, \frac{1}{\eta}\right) \cup \mathcal{R}_{2} \operatorname{diag}\left(\frac{1}{\eta}, \frac{1}{\eta}, \eta^{2}\right)\right)^{\mathrm{rc}} \subset \mathbb{K}^{\mathrm{rc}},
\end{aligned}
$$

because $\lambda_{1}\left(\eta / \lambda_{1}\right)=\eta^{2}(1 \eta)$. Now observe that $\lambda_{1} \lambda_{2} \lambda_{3}=1$, and $\lambda_{i} \in\left[1 / \eta, \eta^{2}\right], i=1,2,3$, imply that $1 / \eta \leq \lambda_{j} \leq$ $\eta / \lambda_{1}, j=2,3$. Thus,

$$
\operatorname{diag}\left(\lambda_{1}, \lambda_{2}, \lambda_{3}\right) \in\left(\mathcal{R}_{1} \operatorname{diag}\left(\lambda_{1}, \frac{\eta}{\lambda_{1}}, \frac{1}{\eta}\right) \cup \mathcal{R}_{1} \operatorname{diag}\left(\lambda_{1}, \frac{1}{\eta}, \frac{\eta}{\lambda_{1}}\right)\right)^{\mathrm{rc}},
$$

and the last set is contained in $\left(\mathcal{R}_{1} \mathbb{K}^{\mathrm{rc}} \cup \mathcal{R}_{1} \mathbb{K}^{\mathrm{rc}}\right)^{\mathrm{rc}}=\mathbb{K}^{\mathrm{rc}}$

We conclude $\mathbb{K}^{\mathrm{pc}} \subset \mathcal{A} \subset \mathbb{K}^{\mathrm{rc}} \subset \mathbb{K}^{\mathrm{pc}}$ and the assertion of the lemma follows. The proof for $\eta<1$, in which case $\eta^{-}=\eta^{2}$ and $\eta^{+}=1 / \eta$, is analogous.

We have not yet been able to compute the quasiconvex envelope of the energy densities (4.3) and (4.4) for $n=3$. The calculation is fundamentally more difficult than in the case $n=2$ because the given densities must now be modified also at points outside $\mathbb{K}^{\mathrm{qc}}$. We make this point more precise in the following proposition.

Proposition 6.2. Assume that $W$ is given by

$$
W(\mathbf{F})=(1-\epsilon) \lambda_{1}^{2}+\lambda_{2}^{2}+\lambda_{3}^{2}-3(1-\epsilon)^{1 / 3},
$$

where $\lambda_{1} \geq \lambda_{2} \geq \lambda_{3}$ are the singular values of $\mathbf{F}$. Let $\mathbb{K}$ be the zero set of $W$,

$$
\mathbb{K}=\bigcup_{\mathbf{e} \in S^{2}} \eta^{2} \mathbf{e} \otimes \mathbf{e}+\frac{1}{\eta}(\mathbf{I}-\mathbf{e} \otimes \mathbf{e})
$$

with $\eta=(1-\epsilon)^{-1 / 6}>1$. Then there exists $\overline{\mathbf{F}} \notin \mathbb{K}^{\mathrm{qc}}$ such that $W^{\mathrm{qc}}(\overline{\mathbf{F}})<W(\overline{\mathbf{F}})$.

Proof. For $\alpha>\eta$, define

$$
\mathbb{K}_{\alpha}=\bigcup_{\mathbf{e} \in S^{2}} \alpha^{2} \mathbf{e} \otimes \mathbf{e}+\frac{1}{\alpha}(\mathbf{I}-\mathbf{e} \otimes \mathbf{e})
$$

and observe that the definition of $\mathbb{K}_{\alpha}^{\mathrm{qc}}$ implies

$$
W^{\mathrm{qc}}(\mathbf{F}) \leq W\left(\operatorname{diag}\left(\alpha^{2}, \frac{1}{\alpha}, \frac{1}{\alpha}\right)\right) \text { for all } \mathbf{F} \in \mathbb{K}_{\alpha}^{\mathrm{qc}} .
$$


Consider the curve in matrix space defined by

$$
t \mapsto \mathbf{F}_{\alpha}(t)=\operatorname{diag}\left(\sqrt{\alpha} t, \frac{1}{\alpha}, \frac{\sqrt{\alpha}}{t}\right), \quad t \in\left[\frac{1}{\alpha \sqrt{\alpha}}, \alpha \sqrt{\alpha}\right],
$$

and notice that, by Proposition 6.1,

$$
\mathbf{F}_{\alpha}(t) \in \mathbb{K}_{\alpha}^{\mathrm{qc}}, \quad \mathbf{F}_{\alpha}(t) \notin \mathbb{K}^{\mathrm{qc}}, \quad t \in\left[\frac{1}{\alpha \sqrt{\alpha}}, \alpha \sqrt{\alpha}\right] .
$$

We claim that, for $\alpha-\eta>0$ small enough,

$$
W\left(\mathbf{F}_{\alpha}(1)\right)>W\left(\mathbf{F}_{\alpha}(\alpha \sqrt{\alpha})\right)=W\left(\operatorname{diag}\left(\frac{1}{\alpha}, \frac{1}{\alpha}, \alpha^{2}\right)\right)
$$

Indeed, the function $f(\alpha):=W\left(\mathbf{F}_{\alpha}(1)\right)-W\left(\mathbf{F}_{\alpha}(\alpha \sqrt{\alpha})\right)$ is continuous and positive in $\alpha=\eta$. Setting $\overline{\mathbf{F}}=\mathbf{F} \alpha(1)$, the proposition follows from (6.2)-(6.4).

\section{Conclusions and discussion}

Let us begin by summarizing our results. In two dimensions, for the prolate case $r>1$, we have found that all possible soft macroscopic deformations are those represented by $2 \times 2$ matrices with determinant equal to 1 and singular values in the interval $\left[r^{-1 / 4}, r^{1 / 4}\right]$. The relaxed energy has the expression $W^{\mathrm{qc}}(\mathbf{F})=\phi\left(\lambda_{1}, \lambda_{2}\right)$ with

$$
\phi\left(\lambda_{1}, \lambda_{2}\right)= \begin{cases}+\infty & \text { if } \lambda_{1} \lambda_{2} \neq 1, \\ 0 & \text { if } \lambda_{1} \lambda_{2}=1, \max \left\{\lambda_{1}, \lambda_{2}\right\} \in\left[1, r^{1 / 4}\right], \\ \mu / 2\left(r^{1 / 2}\left[(1 / r) \max \left\{\lambda_{1}^{2}, \lambda_{2}^{2}\right\}+\min \left\{\lambda_{1}^{2}, \lambda_{2}^{2}\right\}\right]-2\right) & \text { else. }\end{cases}
$$

For the oblate case $r<1$, the possible soft deformations are those represented by $2 \times 2$ matrices with determinant equal to 1 and singular values in the interval $\left[r^{1 / 4}, r^{-1 / 4}\right]$. The relaxed energy has the expression $W_{\mathrm{ob}}^{\mathrm{qc}}(\mathbf{F})=\tilde{\phi}\left(\lambda_{1}, \lambda_{2}\right)$ with

$$
\tilde{\phi}\left(\lambda_{1}, \lambda_{2}\right)= \begin{cases}+\infty & \text { if } \lambda_{1} \lambda_{2} \neq 1, \\ 0 & \text { if } \lambda_{1} \lambda_{2}=1, \max \left\{\lambda_{1}, \lambda_{2}\right\} \in\left[1, r^{-1 / 4}\right], \\ \mu / 2\left(r^{1 / 2}\left[\max \left\{\lambda_{1}^{2}, \lambda_{2}^{2}\right\}+(1 / r) \min \left\{\lambda_{1}^{2}, \lambda_{2}^{2}\right\}\right]-2\right) & \text { else. }\end{cases}
$$

In three dimensions, all possible soft macroscopic deformations are described by $3 \times 3$ matrices with determinant equal to 1 and singular values in the interval $\left[r^{-1 / 6}, r^{1 / 3}\right]$ if $r>1,\left[r^{1 / 3}, r^{-1 / 6}\right]$ if $r<1$.

From a mathematical viewpoint, the natural problem left open by our analysis is that of finding the effective macroscopic energy in three dimensions. In fact, experiments are typically performed by applying biaxial stresses to rubber sheets, thus in a plane stress geometry, rather than in the plane strain geometry for which our two-dimensional formulas would apply. Nevertheless, we hope that our results will be useful to set up numeric simulations of realistic loading histories: as argued, e.g., in [4], algorithms based on relaxed energies may prove rather efficient.

From a physical viewpoint, there are two major areas where our analysis needs refinement. The first issue is the multiplicity of energetically equivalent domain patterns, and our inability to predict their length scales within our current approach. The second issue is the fact that, in reality, deformations paths like the one described in Section 3 are not really "soft" but only "semi-soft" (see [17]).

Regarding the first issue we observe that, as noted in [8], the path of macroscopic deformations (3.1) joining the uniform states $\left(\mathbf{U}_{\mathbf{e}_{3}}, \mathbf{e}_{3}\right)$ and $\left(\mathbf{U}_{\mathbf{e}_{2}}, \mathbf{e}_{2}\right)$ can be resolved microscopically by constructions with the deformation 


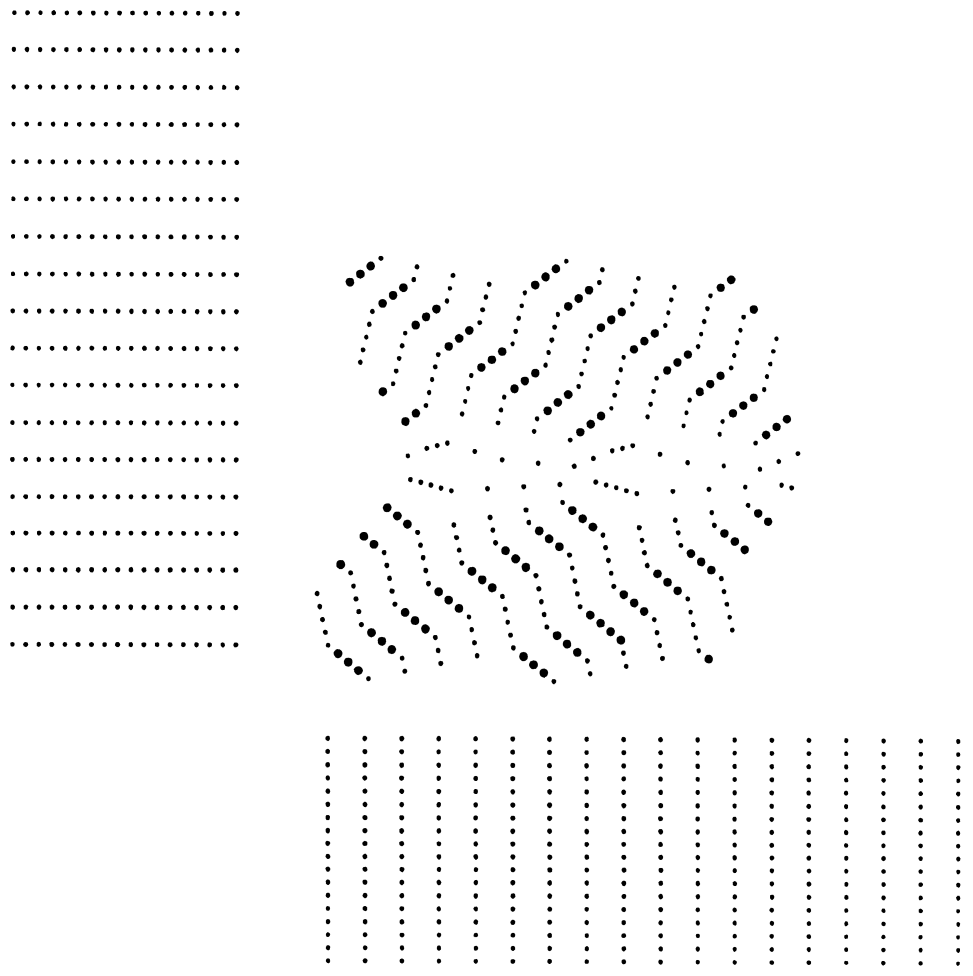

Fig. 5. Alternative domain patterns for the soft deformation path (3.1) from $\mathbf{U}_{\mathbf{e}_{3}}$ (top left) to $\mathbf{U}_{\mathbf{e}_{2}}$ (bottom right).

gradients taking values only ${ }^{11}$ on the two wells $\mathrm{SO}(3) \mathbf{U}_{\mathbf{e}_{3}}$ and $\mathrm{SO}(3) \mathbf{U}_{\mathbf{e}_{2}}$. These alternative domain patterns, shown in Fig. 5, consist of fine layers contained in coarser layers, and are frequently observed in crystalline solids undergoing cubic to tetragonal phase transformations. Although they are geometrically more complicated than the ones actually observed in nematic elastomers, they are energetically equivalent from the point of view of the model analyzed in this paper. In fact, a theory based simply on the free-energy density (2.1) is incapable of predicting the length scale of the energetically optimal domain patterns: the ones shown in Figs. 4 and 5 should be understood as snapshots of infinitely refining sequences of domain arrangements in the spirit of Ball and James [2]. Only in the limit of infinite refinement do these patterns converge to (3.1) (and only in this limit can the two systems of fine layers of Fig. 5 meet through a horizontal interface while being kinematically compatible). Length scales would appear upon introduction of higher order gradients in the free-energy. The most natural such term is the Frank energy, which in the one-constant approximation is $(\kappa / 2) \int_{\Omega}|\nabla \mathbf{n}|^{2}$, with $\kappa$ a positive material constant. A detailed mathematical analysis of the resulting model has not yet been attempted (see, however, [9] for results in this direction).

For what concerns the issue of semi-soft behavior, i.e., the fact that an initial threshold stress needs to be overcome, and then increasing stresses are required to drive the system along the path (3.1), several competing methods of analysis are already available (see $[9,19])$. A consensus on the microscopic mechanisms explaining semi-softness, and on a macroscopic model taking them into account, has yet to be reached. An extensive, quantitative campaign of biaxial stress experiments (as in, e.g., [5]) could help settle controversies, and guide the development of a model capable of describing semi-soft behavior along arbitrary loading paths.

\footnotetext{
$\overline{11}$ This is not true in some transition layers of negligible size and energetic content, see [2]. Examples of such transition layers are the boundary layers required for the piecewise affine deformation in Fig. 4 to meet an affine boundary condition, or the horizontal stripe joining the two systems of slanted fine layers in Fig. 5.
} 


\section{Acknowledgements}

We thank H. Finkelmann, R.D. James, S. Müller, and E.M. Terentjev for useful discussions. This paper is dedicated to J.M. Ball, on the occasion of his fiftieth birthday.

\section{Appendix A. On notation and convexity}

We denote by $\Omega$ a bounded, open, smooth, connected subset of $\mathbb{R}^{n}$, by $\mathbb{M}^{n \times n}$ the set of all $n \times n$ matrices, by $\mathrm{SO}(n)$ the group of proper rotations in $\mathbb{R}^{n}$. For $\mathbf{F} \in \mathbb{M}^{n \times n}, \mathbf{F}^{\mathrm{T}}$ is its transpose, and we call singular values of $\mathbf{F}$ the eigenvalues of $\left(\mathbf{F F}^{\mathrm{T}}\right)^{1 / 2}$. We write $\hat{\mathbf{F}}$ for the vector of all minors of $\mathbf{F}$. For $n=2$, we have $\hat{\mathbf{F}}=(\mathbf{F}, \operatorname{det} \mathbf{F})$; for $n=3$, we have $\hat{\mathbf{F}}=(\mathbf{F}, \operatorname{cof} \mathbf{F}$, $\operatorname{det} \mathbf{F})$, where $\operatorname{det} \mathbf{F}$ is the determinant of $\mathbf{F}$, and $\operatorname{cof} \mathbf{F}$ is the cofactor matrix of $\mathbf{F}$ (if $\mathbf{F}$ is invertible, $\left.\operatorname{cof} \mathbf{F}=(\operatorname{det} \mathbf{F})\left(\mathbf{F}^{-1}\right)^{\mathrm{T}}\right)$. Thus $\hat{\mathbf{F}} \in \mathbb{R}^{N}$, with $N=5$ if $n=2$, and $N=19$ if $n=3$. Finally, we will denote by $\mathbb{R}_{+}^{n}=\left\{\mathbf{x} \in \mathbb{R}^{n}: x_{i} \geq 0, i=1, \ldots, n\right\}$ the positive orthant in $\mathbb{R}^{n}$, and by $S^{n-1}$ the unit sphere in $\mathbb{R}^{n}$.

Let $f: \mathbb{M}^{n \times n} \rightarrow \mathbb{R}$ be a continuous function. We say that

- $f$ is rank-one convex if for each matrix $\mathbf{A} \in \mathbb{M}^{n \times n}$ and each rank-one matrix $\mathbf{B} \in \mathbb{M}^{n \times n}$ the function $t \mapsto$ $f(\mathbf{A}+t \mathbf{B})$ is convex.

- $f$ is quasiconvex if for each matrix $\mathbf{A} \in \mathbb{M}^{n \times n}$ and every smooth function $\mathbf{u}: \Omega \rightarrow \mathbb{R}^{n}$ compactly supported in $\Omega$ the inequality

$$
\int_{\Omega} f(\mathbf{A}) \mathrm{dx} \leq \int_{\Omega} f(\mathbf{A}+\nabla \mathbf{u}(\mathbf{x})) \mathrm{dx}
$$

holds true. The class of quasiconvex functions is independent of $\Omega$.

- $f$ is polyconvex if $f(\mathbf{A})$ is a convex function of the minors of the matrix $\mathbf{A}$, i.e., if $f(\mathbf{A})=G(\hat{\mathbf{A}})$ for some convex function $G: \mathbb{R}^{N} \rightarrow \mathbb{R}$.

It is well known (see [6]) that

$$
f \text { polyconvex } \Rightarrow f \text { quasiconvex } \Rightarrow f \text { rank-one convex. }
$$

We define the rank-one convex envelope $f^{\mathrm{rc}}$ of $f$ by the formula

$$
f^{\mathrm{rc}}(\mathbf{F})=\sup \{g(\mathbf{F}): g \text { rank-one convex, } g \leq f\} .
$$

The quasiconvex envelope $f^{\mathrm{qc}}$ and the polyconvex envelope $f^{\mathrm{pc}}$ of $f$ are defined analogously. It follows from (A.1) and the respective definitions that in general

$$
f^{\mathrm{pc}} \leq f^{\mathrm{qc}} \leq f^{\mathrm{rc}} \leq f
$$

Objective and isotropic free-energies are of particular interest in mechanics. A function $W: \mathbb{M}^{n \times n} \rightarrow \mathbb{R}$ is objective and isotropic if there exists $\Phi: \mathbb{R}^{n} \rightarrow \mathbb{R}$, symmetric in its arguments, such that

$$
W(\mathbf{F})=\Phi\left(v_{1}, \ldots, v_{n}\right),
$$

where $v_{1}, . ., v_{n}$ are the singular values of $\mathbf{F}$. The following results are proved in [1].

Lemma A.1. Let $n \geq 1$. Let $\Phi\left(v_{1}, \ldots, v_{n}\right)$ be a symmetric real-valued function defined on $\mathbb{R}_{+}^{n}$. For $\mathbf{F} \in \mathbb{M}^{n \times n}$ define

$$
W(\mathbf{F})=\Phi\left(v_{1}, \ldots, v_{n}\right)
$$


where $v_{1}, \ldots, v_{n}$ are the singular values of $\mathbf{F}$. Then $W$ is convex on $\mathbb{M}^{n \times n}$ if and only if $\Phi$ is convex and nondecreasing in each variable $v_{i}$.

Lemma A.2. Assume that $U=\left\{\mathbf{F} \in \mathbb{M}^{2 \times 2}: \operatorname{det} \mathbf{F} \in K\right\}$, where $K \subset \mathbb{R}_{+}$is convex. Let

$$
W(\mathbf{F})=\psi\left(v_{1}, v_{2}, v_{1} v_{2}\right),
$$

where $v_{1}, v_{2}$ are the singular values of $\mathbf{F} \in U$, and where $\psi: \mathbb{R}_{+}^{2} \times K \rightarrow \mathbb{R}$ is convex and satisfies

- $\psi\left(x_{1}, x_{2}, \delta\right)=\psi\left(x_{2}, x_{1}, \delta\right)$ for all $x_{1}, x_{2} \in \mathbb{R}_{+}, \delta \in K$,

- $\psi\left(x_{1}, x_{2}, \delta\right)$ is nondecreasing in $x_{1}, x_{2}$.

Then $W$ is polyconvex in $U$.

Let $\mathbb{K} \subset \mathbb{M}^{m \times n}$ be a compact set. We define its rank-one convex hull $\mathbb{K}^{\mathrm{rc}}$ by

$$
\mathbb{K}^{\mathrm{rc}}=\left\{\mathbf{F}: f(\mathbf{F}) \leq \sup _{\mathbf{G} \in \mathbb{K}} f(\mathbf{G}) \forall f: \mathbb{M}^{m \times n} \rightarrow \mathbb{R} \text { rank-one convex }\right\}
$$

The quasiconvex hull $\mathbb{K}^{\mathrm{qc}}$ and the polyconvex hull $\mathbb{K}^{\mathrm{pc}}$ are defined analogously. It follows from (A.1) and the respective definitions that in general

$$
\mathbb{K} \subset \mathbb{K}^{\mathrm{rc}} \subset \mathbb{K}^{\mathrm{qc}} \subset \mathbb{K}^{\mathrm{pc}}
$$

We collect in the lemma below a few elementary properties of rank-one convex hulls which are useful for our calculations.

Lemma A.3. If $\mathbb{K}_{1} \subset \mathbb{K}$ and $\mathbb{K}_{2} \subset \mathbb{K}^{\mathrm{rc}}$, then $\mathbb{K}_{1}^{\mathrm{rc}} \subset \mathbb{K}^{\mathrm{rc}}$ and $\mathbb{K}_{2}^{\mathrm{rc}} \subset \mathbb{K}^{\mathrm{rc}}$. If $\mathbf{Q} \mathbb{K}=\mathbb{K}$ for some $\mathbf{Q} \in \mathrm{SO}(n)$, then $\mathbf{Q} \mathbb{K}^{\mathrm{rc}}=\mathbb{K}^{\mathrm{rc}}$. Similarly, if $\mathbf{Q} \mathbb{K} \mathbf{Q}^{\mathrm{T}}=\mathbb{K}$ then $\mathbf{Q} \mathbb{K}^{\mathrm{rc}} \mathbf{Q}^{\mathrm{T}}=\mathbb{K}^{\mathrm{rc}}$.

Proof. The first two statements follow immediately from the definition of $\mathbb{K}^{\mathrm{rc}}$. To prove the third, assume first that $\mathbf{F} \in \mathbb{K}^{\mathrm{rc}}$, but $\mathbf{Q F} \notin \mathbb{K}^{\mathrm{rc}}$. By definition there exists a rank-one convex function $f$ such that $f(\mathbf{Q F})>\sup _{\mathbb{K}} f$. Let $g(\mathbf{X})=f(\mathbf{Q X})$. Then $g$ is rank-one convex and

$$
g(\mathbf{F})=f(\mathbf{F Q})>\sup _{\mathbb{K}} f=\sup _{\mathbf{Q} \mathbb{K}} f=\sup _{\mathbb{K}} g,
$$

contradicting $\mathbf{F} \in \mathbb{K}^{\mathrm{rc}}$. The remaining statement can be proven similarly.

Our entire analysis rests on the following result proved in [14] (see also [2]).

Lemma A.4. Let $\mathbb{K}=\mathrm{SO}(2) \mathbf{U}_{1} \cup \mathrm{SO}(2) \mathbf{U}_{2}$, where $\mathbf{U}_{1}=b\left(\mathbf{e}_{1} \otimes \mathbf{e}_{1}\right)+a\left(\mathbf{e}_{2} \otimes \mathbf{e}_{2}\right), \mathbf{U}_{2}=a\left(\mathbf{e}_{1} \otimes \mathbf{e}_{1}\right)+b\left(\mathbf{e}_{2} \otimes \mathbf{e}_{2}\right)$, $0<a<b$, and $\left(\mathbf{e}_{1}, \mathbf{e}_{2}\right)$ is the standard basis of $\mathbb{R}^{2}$. Then $\mathbf{F} \in \mathbb{K}^{\mathrm{rc}}=\mathbb{K}^{\mathrm{qc}}=\mathbb{K}^{\mathrm{pc}}$ if and only if

$$
\mathbf{F}=\left(\begin{array}{ll}
y_{1} & y_{2} \\
-y_{2} & y_{1}
\end{array}\right) \mathbf{U}_{1}+\left(\begin{array}{ll}
z_{1} & z_{2} \\
-z_{2} & z_{1}
\end{array}\right) \mathbf{U}_{2}
$$

with $\left(y_{1}^{2}+y_{2}^{2}\right)^{1 / 2}+\left(z_{1}^{2}+z_{2}^{2}\right)^{1 / 2} \leq 1$.

We use repeatedly the following immediate consequence of Lemma A.4.

Lemma A.5. Let $\mathbb{K}=\mathrm{SO}(2) \mathbf{U}_{1} \cup \mathrm{SO}(2) \mathbf{U}_{2}$, where $\mathbf{U}_{1}=b\left(\mathbf{e}_{1} \otimes \mathbf{e}_{1}\right)+a\left(\mathbf{e}_{2} \otimes \mathbf{e}_{2}\right), \mathbf{U}_{2}=a\left(\mathbf{e}_{1} \otimes \mathbf{e}_{1}\right)+b\left(\mathbf{e}_{2} \otimes \mathbf{e}_{2}\right)$, $0<a<b$, and $\left(\mathbf{e}_{1}, \mathbf{e}_{2}\right)$ is the standard basis of $\mathbb{R}^{2}$. Then every diagonal matrix $\mathbf{F}_{\alpha}=\operatorname{diag}(\beta, \alpha)$ such that $\alpha \beta=a b$ and $\alpha \in[a, b]$ belongs to $\mathbb{K}^{\mathrm{rc}}=\mathbb{K}^{\mathrm{qc}}=\mathbb{K}^{\mathrm{pc}}$.

Proof. For $\alpha \in[a, b], \alpha \beta=a b$, we can write $\mathbf{F}_{\alpha}$ as 


$$
\left(\begin{array}{ll}
\beta & 0 \\
0 & \alpha
\end{array}\right)=\left(\begin{array}{ll}
y_{1} & 0 \\
0 & y_{1}
\end{array}\right)\left(\begin{array}{ll}
b & 0 \\
0 & a
\end{array}\right)+\left(\begin{array}{cc}
z_{1} & 0 \\
0 & z_{1}
\end{array}\right)\left(\begin{array}{ll}
a & 0 \\
0 & b
\end{array}\right)
$$

where

$$
y_{1}=\frac{b \beta-a \alpha}{b^{2}-a^{2}} \geq 0, \quad z_{1}=\frac{b \alpha-a \beta}{b^{2}-a^{2}} \geq 0 .
$$

It follows from Lemma A.4 that $\mathbf{F}_{\alpha} \in \mathbb{K}^{\mathrm{rc}}=\mathbb{K}^{\mathrm{qc}}=\mathbb{K}^{\mathrm{pc}}$ because

$$
y_{1}+z_{1}=\frac{\beta+\alpha}{b+a} \leq 1
$$

\section{References}

[1] J.M. Ball, Convexity conditions and existence theorems in nonlinear elasticity, Arch. Rat. Mech. Anal. 63 (1977) $337-403$.

[2] J.M. Ball, R.D. James, Proposed experimental tests of a theory of fine microstructure and the two-well problem, Phil. Trans. Roy. Soc. London A 338 (1992) 389-450.

[3] P. Bladon, E.M. Terentjev, M. Warner, Transitions and instabilities in liquid-crystal elastomers, Phys. Rev. E 47 (1993) R3838-R3840.

[4] C.C. Carstensen, P. Plecháč, Numerical solution of the scalar double-well problem allowing microstructure, Math. Comp. 66 (1997) 997-1026.

[5] C. Chu, Hysteresis and microstructure: a study of biaxial loading on compound twins of copper-aluminum-nickel single crystals, Thesis, University of Minnesota, 1993.

[6] B. Dacorogna, Direct methods in the calculus of variations, Sringer, Berlin, 1989.

[7] P.G. de Gennes, J. Prost, The physics of liquid crystals, Clarendon Press, Oxford, 1993.

[8] A. DeSimone, Energetics of fine domain structures, Ferroelectrics (1999), in press.

[9] H. Finkelmann, I. Kundler, E.M. Terentjev, M. Warner, Critical stripe-domain instability of nematic elastomers, J. Phys. II France 7 (1997) $1059-1069$

[10] M. Hebert, R. Kant, P.G. de Gennes, Dynamics and thermodynamics of artificial muscles based on nematic gels, J. Phys. I France 7 (1997) 909-919.

[11] I. Kundler, H. Finkelmann, Strain induced director reorientation in nematic single crystal elastomers, Makromol. Chem. Rapid Commun. 16 (1995) 679-686.

[12] S. Müller, Variational models for microstructure and phase transitions, Springer Lecture Notes in Mathematics, in press (see also http://www.mis.mpg.de/cgi-bin/lecturenotes.pl).

[13] P. Podio-Guidugli, G. Vergara Caffarelli, Equilibrium phases and layered phase mixtures in elasticity, Math. Mod. Meth. Appl. Sci. 2 (1992) $143-166$.

[14] V. Šverák, On the problem of two wells, in: Microstructure and Phase Transitions, IMA Volumes in Applied Mathematics vol. 54, Springer, Berlin, 1994.

[15] V. Šverák, Rank-one convexity does not imply quasiconvexity, Proc. Roy. Soc. Edinburgh 120 (1992) 185-189.

[16] E. Varley, A. Day, Equilibrium phases of elastic material at uniform temperature and pressure, Arch. Rational Mech. Anal. 22 (1966) 253-269.

[17] M. Warner, New elastic behaviour arising from the unusual constitutive relation of nematic solids, J. Mech. Phys. Solids 47 (1999) $1355-1377$.

[18] M. Warner, E.M. Terentjev, Nematic elastomers — a new state of matter?, Prog. Polym. Sci. 21 (1996) 853-891.

[19] J. Weilepp, H.R. Brand, Director reorientation in nematic-liqui-single-crystal elastomers by external mechanical stress, Europhys. Lett. 34 (1996) 495-500. 